\title{
Correction to: Electrocatalysis, Volume 11, issue 5, September 2020
}

\author{
Springer Nature ${ }^{1}$
}

Published online: 8 February 2021

(c) Springer Science+Business Media, LLC, part of Springer Nature 2021

Correction to: Electrocatalysis (2021) 11(5)

https://doi.org/10.1007/s12678-020-00608-9

https://doi.org/10.1007/s12678-020-00607-w

https://doi.org/10.1007/s12678-020-00609-8

https://doi.org/10.1007/s12678-020-00610-1

https://doi.org/10.1007/s12678-020-00604-z

https://doi.org/10.1007/s12678-020-00611-0

https://doi.org/10.1007/s12678-020-00613-y

https://doi.org/10.1007/s12678-020-00612-z

https://doi.org/10.1007/s12678-020-00615-w

https://doi.org/10.1007/s12678-020-00616-9

The Publisher regrets an error on the cover date year of the September 2020 issue. The cover date year was incorrectly listed as 2021. The correct year should be: 2020 .

The original articles can be found online at https://doi.org/10.1007/s12678-020-00608-9 https://doi.org/10.1007/s12678-020-00607-w https://doi.org/10.1007/s12678-020-00609-8 https://doi.org/10.1007/s12678-020-00610-1 https://doi.org/10.1007/s12678-020-00604-z https://doi.org/10.1007/s12678-020-00611-0 https://doi.org/10.1007/s12678-020-00613-y https://doi.org/10.1007/s12678-020-00612-z https://doi.org/10.1007/s12678-020-00615-w https://doi.org/10.1007/s12678-020-00616-9.

Springer Nature

maryjane.gargar@springer.com

1 New York, United States of America 